\title{
The traditional use of plants to manage candidiasis and related infections in Venda, South Africa
}

\author{
Ndivhaleni A. Masevhea,b, Lyndy J. McGawa, Jacobus N. Eloffa \\ a Phytomedicine Programme, Department of Paraclinical Sciences, Faculty of Veterinary Science, \\ University of Pretoria, Private Bag X04, Onderstepoort 0110, South Africa. \\ ${ }^{b}$ Department of Botany, School of Mathematics and Natural Sciences, University of Venda, Thohoyandou \\ 0950, South Africa \\ Corresponding author: (Tel. / Fax: +27 (0) 12529 8525; E-mail: kobus.eloff@up.ac.za
}

\begin{abstract}
Ethnopharmacological relevance: This paper presents results of an ethnobotanical survey of medicinal plants used for the management of candidiasis and related fungal infections in the Venda area, South Africa.

Materials and Methods: Ethnobotanical data about the uses of plants were gathered from eleven rural traditional healers using semi-structured interviews.

Results: A total of 45 species belonging to 24 different families were identified, of which the dominant family was the Fabaceae with 13 species (28.9\%) followed by the Asteraceae and Solanaceae with 3 species each (6.7 \%). A total of 28 of these plant species (62.2\%) have been shown to have anticandidal activity and 14 species $(31 \%)$ have been recorded for antifungal uses in the literature. Amongst the 45 species recorded, $51 \%$ were trees, $33 \%$ were shrubs, and $16 \%$ were herbs. The most widely used plant species were Acacia caffra, Clerodendrum glabrum, Croton gratissimus, Elaeodendron transvaalense, Faurea saligna, Hippocratea longipetiolata, Osyris lanceolata, Richardia brasiliensis, Schkuhria pinnata, Schotia brachypetala, Spilanthes acmella, Strychnos potatorum, Vangueria infausta subsp. infausta and Withania somnifera. The plant parts mostly used in the therapeutic preparations were roots (27.7\%), bark (23.2\%), and a combination of roots, bark (18.7\%) and leaves (14.3\%). Decoctions (44.4\%), infusions (20\%), macerations $(17.7 \%)$, burning $(11.4 \%)$ and paste $(6.5 \%)$ were used. Most of the herbal remedies were administered orally. The main factors threatening the conservation status of these plants are unsustainable methods of harvesting, logging for firewood, building materials and crafts.

Conclusion: The Venda area is rich in plant diversity and local indigenous knowledge of medicinal plants can play an important role as a model for low cost primary health care. Further studies are in progress to validate the indigenous plants recorded as traditional remedies in this area.
\end{abstract}

Keywords: ethnobotanical survey, candidiasis, Venda, traditional healers, antifungal. 


\section{Graphical abstract}

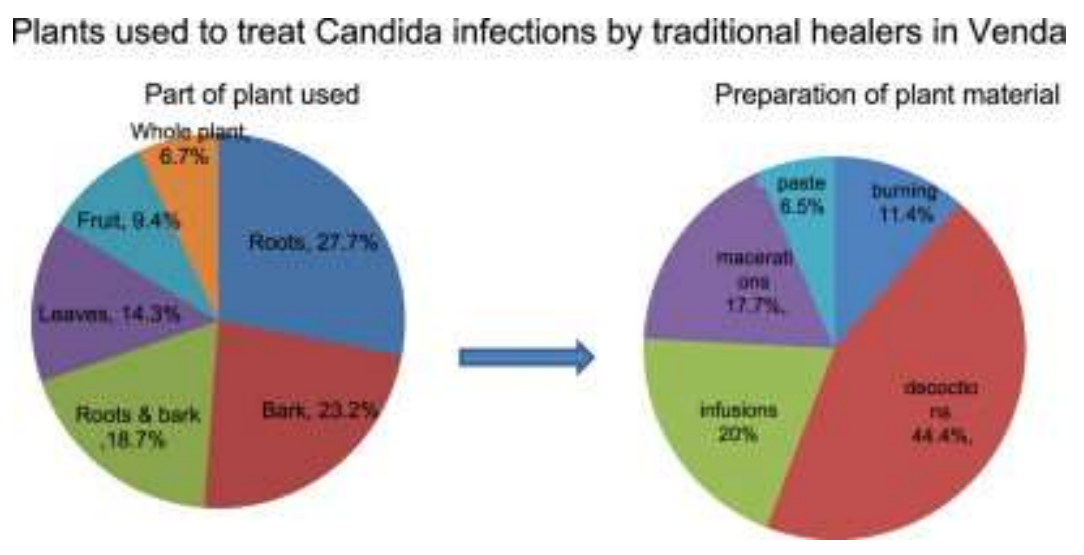

\section{Introduction}

Since ancient times, plants have been used all over the world as unique sources of medicines and constitute the most common human use of biodiversity (Ribeiro et al., 2010). In Africa and in other developing countries many people depend on medicinal plants because they have no access to modern medicines (Runyoro et al., 2006). The dependence on medicinal plants and traditional healers may be attributed to the low proportion of medical doctors to patients in Africa (South Africa 1:1 639; Ethiopia 1:33 000; Kenya 1:7 142; Tanzania 1:33 000; Uganda 1:25 000, Malawi 1:50 000; Mozambique 1:50 000; Swaziland 1:10 000) (Bekalo et al., 2009). In these communities, traditional healers operate closer to the people, taking advantage of the diversity of plant species in such areas to treat various diseases and ailments (Kambizi and Afolayan, 2001).

Herbal medicines have been used to treat many diseases that are obstinate and incurable in other systems of medicine and they are gaining popularity because of several perceived advantages such as fewer side effects, better patient tolerance, relatively lower expense and more ready acceptance due to a long history of use (Vermani and Garg, 2002). However, indigenous knowledge on medicinal plants is being lost at a rapid rate with the increase of modern education, which has led the younger generation not appreciating its traditional values (Zerabruk and Yirga, 2012). This useful information about medicinal plants is also still passed on from one generation to another by oral communication, posing the danger of loss of valuable knowledge (Maregesi et al., 2007). There is still a a need of detailed documentation on the use of medicinal plants in South Africa (Taylor et al. 2001). It is becoming increasingly urgent to document the medicinal use of African plants because of the rapid loss of the natural habitat for some of these plants due to anthropogenic activities (Bisi-Johnson et al., 2010). Thus, there is a need to document the medicinal plants used traditionally before this important knowledge is lost. 
Ethnobotanical studies are important in disclosing locally important plants used by communities in the management of a range of ailments affecting them. There is a wide interest in the use of medicinal plants by Venda people and this has led to several publications (Mahwasane et al., 2013; Mulaudzi et al., 2011 and 2013; Samie et al., 2010; Tshikalange et al., 2005; Mabogo 1990; Arnold and Gulumiam, 1984). In this region, traditional medicine still plays a significant role in the lives of local people, despite recent advances in Western medicine (Meyer et al., 2008). As far as our literature search could ascertain, this is one of the few studies on the medicinal plants claimed to be used to treat candidiasis and related infections.

Candida infections are relatively easy to identify and it is not difficult to see a positive outcome of treatment. There could therefore be a good correlation between traditional use and in vitro efficacy. With the high incidence of HIVIAIDS, about 5.6 million infected people in South Africa (UNAIDS, 2011), candidiasis is a serious challenge to the public health system. The major concern with candidiasis is that it is associated with a mortality rate of $10-49 \%$ in immune compromised patients (Pfaller et al., 2007). Thus, the search for alternative cures from traditional medicine is justified. Therefore, the aim of the study was to investigate and document plants used by local traditional practitioners for the management of candidiasis and related fungal infections

\section{Materials and Methods}

\subsection{Study site and Venda community}

The study took place in four main rural areas of Venda (Mutale, Thohoyandou, Nzhelele and Mashau), Limpopo Province, South Africa (Fig 1). In this region, traditional medicine still plays a significant role in the lives of local people, despite recent advances in Western medicine (Meyer et al., 2008). The region lies in the north-eastern corner of the Soutpansberg and is located between latitudes $22^{\circ} 15^{\prime \prime}$ and $23^{\circ} 45^{\prime \prime} \mathrm{S}$. and longitudes $29^{\circ} 50^{\prime \prime}$ and $31^{\circ} 30^{\prime \prime} \mathrm{E}$. (Lahiff, 1997) with an estimatimation of 1.1 million people (Bornman et al., 2012).Male and female roles are clearly defined, with the men responsible for keeping livestock, ploughing and the building of huts, while the women do most of the harvesting as well as all the domestic duties. Maximum temperatures vary from 25 to $40^{\circ} \mathrm{C}$ in summer and from 22 to $26^{\circ} \mathrm{C}$ in winter. Rainfall is seasonal with $80 \%$ occurring between October and March (Mzezewa et al., 2010. The Venda community is one of the most remote tribes in South Africa with their own language and a distinct culture and knowledge of medicinal plants. They depend on the natural environment for their health care and survival (Mulaudzi et al., 2012). It is an area covered by the north-eastern mountain sourveld vegetation (Acocks, 1988), used in alleviating hunger, for shelters, fuel, artifacts and traditional medicine (Mabogo, 1990). 


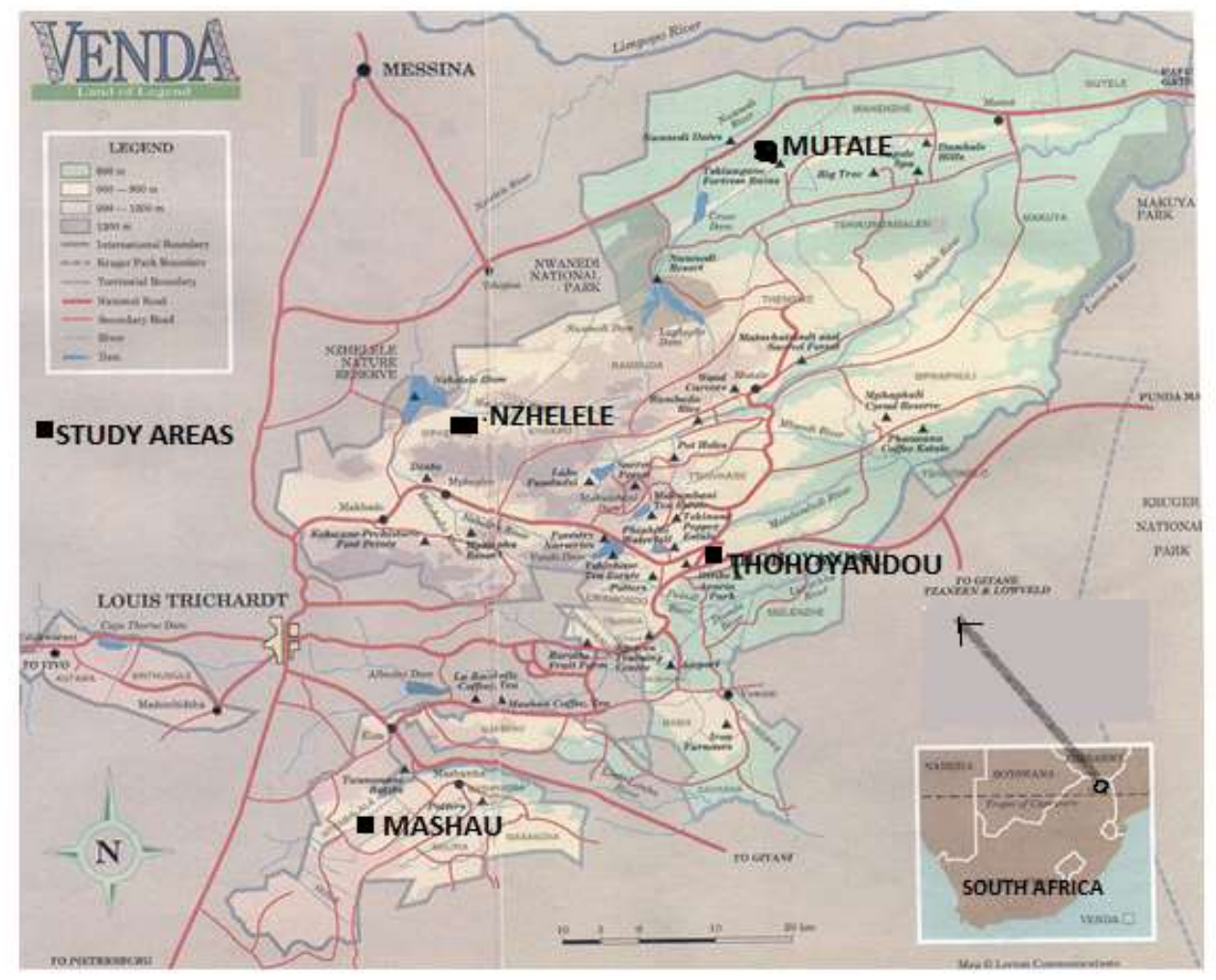

Fig.1. Map of Venda region in Limpopo Province, South Africa

2.2. Interviews with the local traditional healers

Traditional healers were identified in the selected villages after consultation with the headman of each area andpermission was granted by the headman to conduct the study. Main areas visited included Nzhelele, Hamashau, Thohoyandou and Mutale. The first author is of Venda origin, teaches ethnobotany at the University of Venda and because he grew up in the rural environment, speaks the local language and knows all the local customs he was trusted by the traditional healers. The aim of the study was explained to the traditional healers before the interview was conducted, and informed consent was obtained so that they could share their knowledge. Ethnobotanical data were collected using semi-structured interviews in the local language (Tshivenda) and later translated into English. The informants were queried about the symptoms of candidiasis as well as information about the plants they use, including their local names, nature of the plant, plant parts used, methods of preparation, administration techniques, dosage form of the remedy and conservation status of the plants. Personal information was also recorded. A monetary incentive was given to the traditional healers for their time. 


\subsection{Plant collection and preparation of herbarium specimens}

Plants were collected from the wild between September 2010 and June 2011 with the assistance of traditional healers, identified by two botanists from the University of Venda and were authenticated by SANBI (South African National Biodiversity Institute) in Pretoria. Voucher specimens were prepared, deposited at the University of Venda herbarium, South Africa and voucher numbers are recorded in Table 1.

\subsection{Statistical analysis}

Analysis of data was done using inferential and descriptive statistics such as percentages and frequencies. Frequency index (Table 1) was calculated using the following formula:

$: F I=F C / N \times 100$

where $\mathrm{Fl}$ is \% of frequency of citation for one plant species by informants, $\mathrm{FC}$ is the number of informants who cited the use of the plant species, and $\mathrm{N}$ is the total number of informants (11 in this study) (Madikizela et al., 2012).

\section{Results and discussion}

\subsection{Interviews with traditional healers}

Eleven traditional healers were interviewed, namely three males (27.3\%) and eight females (72.7\%), ranging in age from 36 to 69 years. The four age groups $30-40,41-50,51-60$ and $61-70$ were represented by $5,16,52$ and $27 \%$ of the total respectively.Some of them (18\%) received primary school education while $82 \%$ did not have any formal schooling. Nine out of these 11 traditional practitioners were registered with the Vhembe Traditional Healers Association and have membership certificates. The association is recognized by the Department of Health but they are not yet allowed to practice in public health facilities. Nevertheless, $18 \%$ of the traditional healers indicated that occasionally they refer patients to the hospital for check-up after treatment.

According to some traditional healers, candidiasis is known as "Makuma", a Tshivenda word referring to ulcers, which may be either oral or genital. They believe that the ulcers begin in the stomach and move up the alimentary tract until they manifest externally either in the mouth or the genitals. With regard to the causes, there are two schools of thoughts: some believe that it is caused by germs and others believe that it is caused by the transmission of sexually transmitted diseases from one person to another. To ensure that the traditional healers understood the symptoms of candidiasis, we described the symptoms as painful creamy white/red lesions on the angles of the mouth, tongue and also the palate, creamy white discharge and itching of genitals. We also showed pictures of patients suffering from 
the disease.

Males were generally not so keen to participate in the survey even though they were assured that the information was for research purposes only.Many indicated that they could not give away their ancestral knowledge to the researchers, and thus only $27.3 \%$ of the interviewed traditional healers were male. On the other hand females were open, participated freely and even wanted their pharmacopoeia to be evaluated for efficacy and toxicity. The fact that $52 \%$ of traditional healers interviewed ranged in age from 51-60 years indicated the wealth of indigenous knowledge gained in this survey as more experienced practitioners could be assumed to have greater knowledge than younger practitioners . On the other hand, this shows an urgent need for documenting more ethnobotanical data in the area because the future of indigenous knowledge is being threatened by remaining largely in the hands of older people. In most of the meetings with the traditional practitioners, there were very few young traditional practitioners, howeer there was no enquiry as to how many were still undergoing training under the established traditional practitioners.

\subsection{Plant species and their families}

Fourty five I plant species claimed to be used by local traditional healers to treat candidiasis and related infections were recorded. Table 1 shows the botanical name, family, Venda common name, voucher number, frequency index, part used, method of preparation, route of administration and reported ethnomedicinal uses of the herbal remedy. Plant species were distributed among 41 genera and 24 different plant families. The Fabaceae wasthe most represented with 12 plant species, followed by the Asteraceae and Solanaceae (3 plant species each), Apocynaceae, Ebenaceae, Celastraceae, Loganiaceae, Rubiaceae (2 plant species each), and the rest of the families were represented by one plant species each. Frequent use of members of the Fabaceae family has been documented by several surveys in Nigeria (Offiah et al, 2011); Ethiopia (Lulekal et al., 2008), and South Africa (BisiJohnson et al., 2010). This is not surprising because it is the third largest family of angiosperm plants with approximately 730 genera and over 19400 species worldwide (Arabi and Sardari, 2010). 
Table 1. Plants and procedures used to treat candidal infections by traditional practitioners.

\begin{tabular}{|c|c|c|c|c|c|c|c|c|c|c|}
\hline $\begin{array}{l}\text { Botanical name } \\
\text { Family }\end{array}$ & $\begin{array}{l}\text { Local } \\
\text { name }\end{array}$ & $\begin{array}{l}\text { Vou } \\
\text { cher } \\
\text { no }\end{array}$ & $\begin{array}{l}\mathrm{Ha} \\
\text { bit }\end{array}$ & $\begin{array}{l}\text { Fre } \\
\text { que } \\
\text { ncy } \\
\text { inde } \\
x\end{array}$ & $\begin{array}{l}\mathrm{Pa} \\
\text { it } \\
\text { us } \\
\text { ed }\end{array}$ & Method of preparation & Route of administration & $\begin{array}{l}\text { anti-Candida } \\
\text { activity/active } \\
\text { constituents }\end{array}$ & $\begin{array}{l}\text { Relevant reported } \\
\text { ethnomedicinal uses }\end{array}$ & $\begin{array}{l}\text { Previous report on other } \\
\text { ethnomedicinal uses }\end{array}$ \\
\hline $\begin{array}{l}\text { Acacia karoo } \\
\text { Hayne. } \\
\text { Fabaceae }\end{array}$ & Muunga & $\begin{array}{l}\mathrm{Rn} \\
34\end{array}$ & $\mathrm{~T}$ & 9 & $\begin{array}{l}\mathrm{r} \\
\mathrm{b}\end{array}$ & $\begin{array}{l}\text { Roots and stem bark } \\
\text { are boiled together }\end{array}$ & $\begin{array}{l}\text { Mouthwash or applied to } \\
\text { genitals, } 2 x \text { per day for a } \\
\text { week }\end{array}$ & (Mulaudzi et al., 2011) & No report & $\begin{array}{l}\text { Venereal diseases, diarrhoea } \\
\text { (Mabogo, 1990) }\end{array}$ \\
\hline $\begin{array}{l}\text { Acacia caffra } \\
\text { (Thunb) Wild. } \\
\text { Fabaceae }\end{array}$ & $\begin{array}{l}\text { Murovha } \\
\text { mbado }\end{array}$ & $\begin{array}{l}\mathrm{Rn} \\
40\end{array}$ & $\mathrm{~T}$ & 45 & I & $\begin{array}{l}\text { Dried, burnt and mixed } \\
\text { with } \\
\text { animal fats }\end{array}$ & $\begin{array}{l}\text { Applied directly on mouth } \\
\text { ulcers or vaginal ulcers }\end{array}$ & No report & No report & $\begin{array}{l}\text { Blood cleansing, abdominal } \\
\text { disorder (Venter and Venter, } \\
\text { 1996) }\end{array}$ \\
\hline $\begin{array}{l}\text { Amaranthus } \\
\text { spinosus L. } \\
\text { Amaranthaceae }\end{array}$ & $\begin{array}{l}\text { Tshithavh } \\
\text { amisis }\end{array}$ & Rn 5 & $\mathrm{H}$ & 18 & I & $\begin{array}{l}\text { add fresh leaves in hot } \\
\text { water }\end{array}$ & $\begin{array}{l}\text { Oral, } 1 / 2 \text { cup of the infusion } \\
\text { is taken } 3 x \text { a day }\end{array}$ & No report & No report & $\begin{array}{l}\text { Analgesic, laxative, piles (Kumar } \\
\text { et al., 2011) }\end{array}$ \\
\hline $\begin{array}{l}\text { Burkea africana } \\
\text { Hook. } \\
\text { Fabaceae }\end{array}$ & Mufhulu & $\begin{array}{l}\mathrm{Rn} \\
24\end{array}$ & $\mathrm{~T}$ & 9 & $\begin{array}{l}\text { se } \\
r\end{array}$ & $\begin{array}{l}\text { Dried roots, seeds are } \\
\text { ground up and then } \\
\text { boiled }\end{array}$ & $\begin{array}{l}\text { Oral, taken } 2 x \text { a day, } \\
\text { overdose causes } \\
\text { diarrhoea }\end{array}$ & (Steenkamp et al., 2007) & No report & $\begin{array}{l}\text { Herpes simplex (Chisembu, } \\
\text { 2010), dysentery (Grant and } \\
\text { Thomas, 2000) }\end{array}$ \\
\hline $\begin{array}{l}\text { Carica papaya L. } \\
\text { Caricaceae }\end{array}$ & $\begin{array}{l}\text { Mupapaw } \\
\text { e }\end{array}$ & $\operatorname{Rn} 9$ & $\mathrm{~T}$ & 36 & $\mathrm{r}$ & $\begin{array}{l}\text { Grind roots of } C \text {. } \\
\text { papaya and } P \text {. guava, } \\
\text { mixed into hot water }\end{array}$ & $\begin{array}{l}\text { Oral, cup is taken } 3 x \text { a day } \\
\text { or douching }\end{array}$ & (Runyoro et al., 2006) & $\begin{array}{l}\text { Vaginal candidiasis } \\
\text { (Runyoro et al., 2006) }\end{array}$ & $\begin{array}{l}\text { Infectious diseases (Magassouba } \\
\text { et al., 2007), cancer (Otsuki et al., } \\
\text { 2010) }\end{array}$ \\
\hline $\begin{array}{l}\text { Carpobrotus edulis } \\
\text { ( L. ) L.Bolus } \\
\text { Mesembryanthema } \\
\text { ceae }\end{array}$ & Lutele & $\begin{array}{l}\mathrm{Rn} \\
32\end{array}$ & S & 9 & $b$ & $\begin{array}{l}\text { Grind dry bark into fine } \\
\text { powder, boil for } 20-30 \\
\text { min }\end{array}$ & $\begin{array}{l}\text { Oral, as mouth wash, } 3 x \\
\text { per day for } 1 \text { week }\end{array}$ & (Motsei et al., 2003) & $\begin{array}{l}\text { Thrush (Thring and } \\
\text { Weitz, 2006) }\end{array}$ & $\begin{array}{l}\text { Diarrhoea (Bisi-Johnson et al., } \\
\text { 2010) }\end{array}$ \\
\hline $\begin{array}{l}\text { Cissampelos } \\
\text { torulosa E.Mey. } \\
\text { Menispermaceae }\end{array}$ & $\begin{array}{l}\text { Lukandulu } \\
\text { lo }\end{array}$ & $\begin{array}{l}\mathrm{Rn} \\
13\end{array}$ & $\mathrm{~T}$ & 27 & $b$ & $\begin{array}{l}\text { Grind dry bark into fine } \\
\text { powder, boil in water }\end{array}$ & Oral, gargle or douche & No report & No report & $\begin{array}{l}\text { Syphilis, toothache (De Wet and } \\
\text { Van Wyk, 2008) }\end{array}$ \\
\hline $\begin{array}{l}\text { Clerodendrum } \\
\text { glabrum E. Mey, } \\
\text { Verbenaceae }\end{array}$ & $\begin{array}{l}\text { Munkhatin } \\
\text { gwe }\end{array}$ & $\begin{array}{l}\mathrm{Rn} \\
46\end{array}$ & S & 64 & $\begin{array}{l}\mathrm{R} \\
\mathrm{b}\end{array}$ & $\begin{array}{l}\text { Dried, pulverized and } \\
\text { then } \\
\text { boiled in water }\end{array}$ & $\begin{array}{l}1 / 4 \text { cup of decoction is } \\
\text { taken orally } 3 x \text { per day }\end{array}$ & No report & $\begin{array}{l}\text { Oral ulcers (Mabogo, } \\
\text { 1990) }\end{array}$ & $\begin{array}{l}\text { Diarrhoea (Bisi-Johnson et al., } \\
\text { 2010), Coughs (McGaw et al., } \\
\text { 2008) }\end{array}$ \\
\hline $\begin{array}{l}\text { Croton gratissimus } \\
\text { Burch. } \\
\text { Euphorbiaceae }\end{array}$ & $\begin{array}{l}\text { Mafunyun } \\
\text { gule }\end{array}$ & $\begin{array}{l}\mathrm{Rn} \\
38\end{array}$ & $S$ & 55 & $b$ & $\begin{array}{l}\text { Fresh bark is crushed, } \\
\text { soaked in hot water or } \\
\text { boiled in water } \\
\text { for few minutes }\end{array}$ & $\begin{array}{l}\text { Cupful infusion or } \\
\text { decoction is drunk orally } \\
2 x \text { per day after meals. }\end{array}$ & $\begin{array}{l}\text { (van Vuuren and Naidoo, } \\
\text { 2010) }\end{array}$ & No report & $\begin{array}{l}\text { Syphilis, earache (van Vuuren } \\
\text { and Viljoen, 2008), pneumonia } \\
\text { (McGaw et al., 2008) }\end{array}$ \\
\hline $\begin{array}{l}\text { Diospyros } \\
\text { whyteana F.White, } \\
\text { Ebenaceae }\end{array}$ & $\begin{array}{l}\text { Munya } \\
\text { vhili }\end{array}$ & $\operatorname{Rn} 6$ & $\mathrm{~T}$ & 27 & I & $\begin{array}{l}\text { Dried leaves are put in } \\
\text { hot water to make tea }\end{array}$ & $\begin{array}{l}\text { Infusion is used as a } \\
\text { douche }\end{array}$ & $\begin{array}{l}\text { Isodiospyrin (Singh et al., } \\
\text { 2012) }\end{array}$ & No report & $\begin{array}{l}\text { Dysmenorrhoea, rash } \\
\text { (Steenkamp, 2003) }\end{array}$ \\
\hline $\begin{array}{l}\text { Diospyros } \\
\text { mespiliformis } \\
\text { Hochst. ex A.DC. } \\
\text { Ebenaceae }\end{array}$ & Musuma & $\begin{array}{l}\mathrm{Rn} \\
31\end{array}$ & $\mathrm{~T}$ & 36 & $f$ & $\begin{array}{l}\text { Crush raw fruit and add } \\
\text { little water }\end{array}$ & $\begin{array}{l}\text { Infusion is used as mouth } \\
\text { wash or douche } 3 x \text { per } \\
\text { day }\end{array}$ & $\begin{array}{l}\text { Isodiospyrin (Singh et al., } \\
\text { 2012) }\end{array}$ & $\begin{array}{l}\text { Fungal infections } \\
\text { (Mabogo, 1990) }\end{array}$ & $\begin{array}{l}\text { Diarrhoea (Mahwasane et al., } \\
\text { 2013), } \\
\text { hypertension (Adamu et al., 2005) }\end{array}$ \\
\hline
\end{tabular}

Table 1 cont... 


\begin{tabular}{|c|c|c|c|c|c|c|c|c|c|c|}
\hline $\begin{array}{l}\text { Botanical name } \\
\text { Family }\end{array}$ & Local name & $\begin{array}{l}\text { Vou } \\
\text { cher } \\
\text { no }\end{array}$ & $\begin{array}{l}\mathrm{H} \\
\mathrm{a} \\
\mathrm{bi} \\
\mathrm{t}\end{array}$ & $\begin{array}{l}\text { Freq } \\
\text { uenc } \\
y \\
\text { inde } \\
x \\
\end{array}$ & $\begin{array}{l}\text { Par } \\
\mathrm{t} \\
\text { us } \\
\text { ed }\end{array}$ & Method of preparation & Route of administration & $\begin{array}{l}\text { Proven anti- } \\
\text { Candida } \\
\text { activity/active } \\
\text { constituents }\end{array}$ & $\begin{array}{l}\text { Relevant reported } \\
\text { ethnomedicinal } \\
\text { uses }\end{array}$ & $\begin{array}{l}\text { Previous report on other } \\
\text { ethnomedicinal uses }\end{array}$ \\
\hline $\begin{array}{l}\text { Dodonaea } \\
\text { angustifolia L.f. } \\
\text { Sapindaceae }\end{array}$ & $\begin{array}{l}\text { Muthathavh } \\
\text { ana }\end{array}$ & $\begin{array}{l}\mathrm{Rn} \\
26\end{array}$ & $S$ & 18 & I & $\begin{array}{l}\text { Fresh leaves are } \\
\text { crushed and macerated } \\
\text { in water. It is used } \\
\text { together with the bark of } \\
\text { Dovyalis zeyheri }\end{array}$ & $\begin{array}{l}\text { Maceration is taken orally, } \\
1 / 2 \text { a cup } 2 x \\
\text { per day }\end{array}$ & (Motsei et al., 2003) & $\begin{array}{l}\text { Oral candidiasis (Patel } \\
\text { and Coogan, 2008) }\end{array}$ & $\begin{array}{l}\text { Pneumonia, TB (van Wyk and } \\
\text { Gericke, 2000) } \\
\text { rhinitis, piles (Vermani and Garg, } \\
\text { 2002) }\end{array}$ \\
\hline $\begin{array}{l}\text { Elaeodendron } \\
\text { transvaalense } \\
\text { (Burtt Davy), } \\
\text { Celastraceae }\end{array}$ & $\begin{array}{l}\text { Mukuvhaz } \\
\text { wivhi }\end{array}$ & $\begin{array}{l}\mathrm{Rn} \\
41\end{array}$ & $\mathrm{~T}$ & 73 & $r$ & $\begin{array}{l}\text { Dried, powdered and } \\
\text { boiled in water }\end{array}$ & $\begin{array}{l}1 / 2 \text { cup of decoction is } \\
\text { drunk } 3 x \text { per day }\end{array}$ & (Samie et al., 2010) & $\begin{array}{l}\text { Candidiasis (Bessong } \\
\text { et al., 2005) }\end{array}$ & $\begin{array}{l}\text { Stomachache, fever (Drewes et al., } \\
\text { 1991), } \\
\text { dysmenorrhoea (Steenkamp, 2003) }\end{array}$ \\
\hline $\begin{array}{l}\text { Elephantorrhiza } \\
\text { burkei Benth, } \\
\text { Fabaceae }\end{array}$ & $\begin{array}{l}\text { Tshisevhuf } \\
\text { a }\end{array}$ & $\begin{array}{l}\mathrm{Rn} \\
17\end{array}$ & $S$ & 18 & $b$ & $\begin{array}{l}\text { Grind dry bark into fine } \\
\text { powder }\end{array}$ & $\begin{array}{l}\text { Topical application, } 2 x \text { per } \\
\text { day }\end{array}$ & $\begin{array}{l}\text { (Mulaudzi et al., } \\
2011\}\end{array}$ & No report & $\begin{array}{l}\text { Venereal diseases, aphrodisiac } \\
\text { (Mabogo, 1990), } \\
\text { abortifacient (Steenkamp, 2003) }\end{array}$ \\
\hline $\begin{array}{l}\text { Erythrina } \\
\text { lysistemon Hutch. } \\
\text { Fabaceae }\end{array}$ & Muvhale & $\begin{array}{l}\mathrm{Rn} \\
27\end{array}$ & $\mathrm{~T}$ & 36 & $\begin{array}{l}\mathrm{r} \\
\mathrm{st} \\
\mathrm{b}\end{array}$ & $\begin{array}{l}\text { Grind up roots and stem } \\
\text { bark and boil } \\
\text { for } 1 \mathrm{~h}\end{array}$ & $\begin{array}{l}\text { Mouth wash or douche, } 2 x \\
\text { per day for a week }\end{array}$ & (Motsei et al., 2003) & No report & $\begin{array}{l}\text { Wounds, arthritis (van Wyk and } \\
\text { Gericke, 2000) } \\
\text { oedema (Mahwasane et al., 2013) }\end{array}$ \\
\hline $\begin{array}{l}\text { Faurea saligna } \\
\text { Harv. Proteaceae }\end{array}$ & Mutango & $\begin{array}{l}\mathrm{Rn} \\
44\end{array}$ & $\mathrm{~T}$ & 64 & $\mathrm{~b}$ & $\begin{array}{l}\text { Dried, powdered and } \\
\text { soaked in warm } \\
\text { water for at least } 30 \mathrm{~min}\end{array}$ & $\begin{array}{l}\text { The infusion is used as a } \\
\text { douche for vaginal ulcers }\end{array}$ & No report & No report & $\begin{array}{l}\text { Diarrhoea (Hamill et al., 2000), } \\
\text { epilepsy (Stafford et al., 2008) }\end{array}$ \\
\hline $\begin{array}{l}\text { Ficus carica L. } \\
\text { Moraceae }\end{array}$ & Muhuyu & $\operatorname{Rn} 3$ & $\mathrm{~T}$ & 27 & $f$ & $\begin{array}{l}\text { Latex is collected from } \\
\text { fig's raw fruit, crushed } \\
\text { leaves and some water } \\
\text { added }\end{array}$ & $\begin{array}{l}\text { Mixture is used as mouth } \\
\text { wash/douche } 3 x \text { per day }\end{array}$ & (Aref et al., 2010) & $\begin{array}{l}\text { Sore throat (Jeong et } \\
\text { al., 2009) }\end{array}$ & Eyesore, diabetes (Aref et al., 2010) \\
\hline $\begin{array}{l}\text { Hippocratea } \\
\text { longipetiolata } \\
\text { Oliv.Celastraceae }\end{array}$ & Mutshaliri & $\begin{array}{l}\mathrm{Rn} \\
37\end{array}$ & $S$ & 55 & $r, b$ & $\begin{array}{l}\text { Used in combination } \\
\text { with few leaves } \\
\text { of } 0 \text {. rochetiana, boiled } \\
\text { in water }\end{array}$ & $\begin{array}{l}\text { A cupful decoction is } \\
\text { drunk orally } 3 x \text { per day. }\end{array}$ & No report & No report & $\begin{array}{l}\text { Invocation of ancestors } \\
\text { (Mabogo,1990) }\end{array}$ \\
\hline $\begin{array}{l}\text { Knowltonia } \\
\text { bracteata Harv. } \\
\text { Ranunculaceae }\end{array}$ & $\begin{array}{l}\text { Thauyakho } \\
\text { mba }\end{array}$ & $\begin{array}{l}\mathrm{Rn} \\
18\end{array}$ & $S$ & 18 & r & $\begin{array}{l}\text { Roots are pulverized } \\
\text { and soaked in } \\
\text { water for at least two } \\
\text { days. }\end{array}$ & $\begin{array}{l}\text { Cupful of the maceration } \\
\text { is taken } 3 x \text { per day }\end{array}$ & $\begin{array}{l}\text { (Buwa and van } \\
\text { Staden, 2006) }\end{array}$ & No report & $\begin{array}{l}\text { Sexually transmitted diseases } \\
\text { (Buwa and van Staden, 2006) }\end{array}$ \\
\hline $\begin{array}{l}\text { Osyris lanceolata } \\
\text { Hochst. \& Steud. } \\
\text { Santalaceae }\end{array}$ & Mpeta & $\begin{array}{l}\mathrm{Rn} \\
45\end{array}$ & $S$ & 64 & $b$ & $\begin{array}{l}\text { Grind bark into fine } \\
\text { powder and } \\
\text { then boil in water }\end{array}$ & $\begin{array}{l}\text { Oral, cup of decoction } \\
\text { is taken } 2 x \text { per day }\end{array}$ & $\begin{array}{l}\text { (Mulaudzi et al., } \\
\text { 2011) }\end{array}$ & No report & Ringworm (Muthee et al.,2011) \\
\hline $\begin{array}{l}\text { Ozoroa engleri } \\
\text { R.Fern. \& A.Fern. } \\
\text { Anacardiaceae }\end{array}$ & $\begin{array}{l}\text { Mudumbu } \\
\text { la }\end{array}$ & $\operatorname{Rn} 8$ & $\mathrm{~T}$ & 27 & $r$ & $\begin{array}{l}\text { Grind dry roots and boil } \\
\text { for } 1 \mathrm{~h} \text {, }\end{array}$ & $\begin{array}{l}\text { Decoction is used as } \\
\text { mouthwash or douche }\end{array}$ & No report & No report & $\begin{array}{l}\text { Sexually transmitted infections } \\
\text { (de Wet et al., 2012) }\end{array}$ \\
\hline
\end{tabular}


Table 1. cont....

\begin{tabular}{|c|c|c|c|c|c|c|c|c|c|c|}
\hline $\begin{array}{l}\text { Botanical name } \\
\text { Family }\end{array}$ & Local name & $\begin{array}{l}\text { Vou } \\
\text { cher } \\
\text { no }\end{array}$ & $\begin{array}{l}\mathrm{H} \\
\mathrm{a} \\
\mathrm{bi} \\
\mathrm{t}\end{array}$ & $\begin{array}{l}\text { Freq } \\
\text { uenc } \\
y \\
\text { inde } \\
x\end{array}$ & $\begin{array}{l}\text { Par } \\
t \\
\text { us } \\
\text { ed }\end{array}$ & Method of preparation & Route of administration & $\begin{array}{l}\text { Proven anti-Candida } \\
\text { activity/active chemical } \\
\text { constituents }\end{array}$ & $\begin{array}{l}\text { Relevant reported } \\
\text { ethnomedicinal uses }\end{array}$ & $\begin{array}{l}\text { Previous report on other } \\
\text { ethnomedicinal uses }\end{array}$ \\
\hline $\begin{array}{l}\text { Pappea capensis } \\
\text { Sond. \& Harv. } \\
\text { Sapindaceae }\end{array}$ & Murodolo & $\begin{array}{l}\mathrm{Rn} \\
30\end{array}$ & $\mathrm{~T}$ & 9 & I & $\begin{array}{l}\text { Fresh leaves are } \\
\text { pounded in some } \\
\text { water and filtered }\end{array}$ & $\begin{array}{l}\text { Juice is used as douche } \\
\text { on vaginal ulcers }\end{array}$ & (Mulaudzi et al., 2011) & No report & $\begin{array}{l}\text { Aphrodisiac, venereal } \\
\text { diseases (Hutchings et } \\
\text { al., 1996) }\end{array}$ \\
\hline $\begin{array}{l}\text { Peltophorum } \\
\text { africanum Sond. } \\
\text { Fabaceae }\end{array}$ & Musese & $\begin{array}{l}\mathrm{Rn} \\
15\end{array}$ & $\mathrm{~T}$ & 36 & $b$ & $\begin{array}{l}\text { Dry bark is soaked in } \\
\text { water for } \\
\text { at least two days }\end{array}$ & $\begin{array}{l}\text { Infusion is taken orally, } 1 / 2 \\
\text { cup } 3 \text { times per day }\end{array}$ & $\begin{array}{l}\text { (Steenkamp et al., } \\
\text { 2007) }\end{array}$ & $\begin{array}{l}\text { Sore throat (Bessong } \\
\text { et al., 2005) }\end{array}$ & $\begin{array}{l}\text { Diarrhoea (McGaw et } \\
\text { al., 2008), Venereal } \\
\text { diseases (de Wet et al., } \\
\text { 2012) }\end{array}$ \\
\hline $\begin{array}{l}\text { Piper capense L.f. } \\
\text { Piperaceae }\end{array}$ & Mulilwe & $\begin{array}{l}\mathrm{Rn} \\
21\end{array}$ & $S$ & 18 & $b$ & $\begin{array}{l}\text { Grind dry bark into fine } \\
\text { powder } \\
\text { and boil }\end{array}$ & $\begin{array}{l}\text { Oral, } 1 \text { cup taken } 3 x \text { per } \\
\text { day }\end{array}$ & $\begin{array}{l}\text { (Steenkamp et al., } \\
\text { 2007) }\end{array}$ & $\begin{array}{l}\text { Sore throat (Mabogo, } \\
1990)\end{array}$ & $\begin{array}{l}\text { Diarrhoea, cough } \\
\text { (Chahal et al., 2011), } \\
\text { Ulcers, fever (Obi et al., } \\
\text { 2002) }\end{array}$ \\
\hline $\begin{array}{l}\text { Pterocarpus } \\
\text { rotundifolius } \\
\text { Druce, } \\
\text { Fabaceae }\end{array}$ & Muataha & $\begin{array}{l}\mathrm{Rn} \\
29\end{array}$ & $\mathrm{~T}$ & 9 & $\begin{array}{l}\text { st } \\
b\end{array}$ & $\begin{array}{l}\text { Grind bark into fine } \\
\text { powder } \\
\text { and pour into boiling } \\
\text { water }\end{array}$ & $\begin{array}{l}\text { Oral, quarter of a cup } \\
\text { taken } 2 x \text { per day }\end{array}$ & No report & No report & $\begin{array}{l}\text { Sore eyes (Venter and } \\
\text { Venter, 1996) }\end{array}$ \\
\hline $\begin{array}{l}\text { Richardia } \\
\text { brasiliensis } \\
\text { Gomes, } \\
\text { Rubiaceae }\end{array}$ & Mulegere & $\begin{array}{l}\mathrm{Rn} \\
14\end{array}$ & $\mathrm{H}$ & 45 & $\begin{array}{l}W \\
p\end{array}$ & $\begin{array}{l}\text { Dried, burnt, pinch } \\
\text { mixed with } \\
\text { animal fats }\end{array}$ & $\begin{array}{l}\text { Applied directly on mouth } \\
\text { ulcers or vaginal ulcers }\end{array}$ & (Adekunle, 2000) & No report & $\begin{array}{l}\text { Diabetes, anti-emetic } \\
\text { (Pinto et al., 2008) }\end{array}$ \\
\hline $\begin{array}{l}\text { Rinorea } \\
\text { Angustifolia Baill, } \\
\text { Violaceae }\end{array}$ & $\begin{array}{l}\text { Mafambab } \\
\text { orile }\end{array}$ & $\begin{array}{l}\mathrm{Rn} \\
28\end{array}$ & $S$ & 18 & $r$ & $\begin{array}{l}\text { Roots are boiled for at } \\
\text { least } 1 \mathrm{~h}, \\
\text { inappropriate } \\
\text { preparation may cause } \\
\text { stomach-ache }\end{array}$ & $\begin{array}{l}\text { Oral, } 1 / 2 \text { a cup is taken } 2 x \\
\text { per day }\end{array}$ & No report & No report & No report \\
\hline $\begin{array}{l}\text { Schotia } \\
\text { Brachypetala } \\
\text { Sond. } \\
\text { Fabaceae }\end{array}$ & Mununzu & $\begin{array}{l}\mathrm{Rn} \\
39\end{array}$ & $\mathrm{~T}$ & 55 & $r, b$ & $\begin{array}{l}\text { Roots and barks are } \\
\text { ground together, boiled } \\
\text { in water }\end{array}$ & $\begin{array}{l}\text { A cupful decoction is taken } \\
\text { orally } 3 x \text { per day after } \\
\text { meals }\end{array}$ & (Samie et al., 2010) & No report & $\begin{array}{l}\text { Dysentery, diarrhoea } \\
\text { (McGaw et al.,2000), } \\
\text { ulcers (Venter and } \\
\text { Venter, 1996) }\end{array}$ \\
\hline $\begin{array}{l}\text { Schkuhria pinnata } \\
\text { (Lam.) Kuntze ex } \\
\text { Thell, Asteraceae }\end{array}$ & Luswielo & $\begin{array}{l}\mathrm{Rn} \\
35\end{array}$ & $\mathrm{H}$ & 73 & wp & $\begin{array}{l}\text { Dried, powdered and } \\
\text { boiled } \\
\text { in water }\end{array}$ & $\begin{array}{l}\text { A cupful of decoction is } \\
\text { drunk } 3 x \text { per day. Also } \\
\text { used to treat HIVIAIDS }\end{array}$ & No report & No report & $\begin{array}{l}\text { Diabetes, oedema } \\
\text { (Mahwasane et al., } \\
\text { 2013) }\end{array}$ \\
\hline
\end{tabular}


Table 1. cont....

\begin{tabular}{|c|c|c|c|c|c|c|c|c|c|c|}
\hline $\begin{array}{l}\text { Botanical name } \\
\text { Family }\end{array}$ & Local name & $\begin{array}{l}\text { Vou } \\
\text { cher } \\
\text { no }\end{array}$ & $\begin{array}{l}\mathrm{H} \\
\mathrm{a} \\
\mathrm{bi} \\
\mathrm{t}\end{array}$ & $\begin{array}{l}\text { Frequ } \\
\text { ency } \\
\text { index }\end{array}$ & $\begin{array}{l}\mathrm{P} \\
\text { ar } \\
\mathrm{t} \\
\mathrm{u} \\
\mathrm{s} \\
\mathrm{e} \\
\mathrm{d}\end{array}$ & Method of preparation & Route of administration & $\begin{array}{l}\text { Proven anti-Candida } \\
\text { activity/active chemical } \\
\text { constituents }\end{array}$ & $\begin{array}{l}\text { Relevant reported } \\
\text { ethnomedicinal uses }\end{array}$ & $\begin{array}{l}\text { Previous report on } \\
\text { other ethnomedicinal } \\
\text { uses }\end{array}$ \\
\hline $\begin{array}{l}\text { Senna auriculata } \\
\text { Roxb. } \\
\text { Fabaceae }\end{array}$ & $\begin{array}{l}\text { Muduwisha } \\
\text { ngo }\end{array}$ & $\begin{array}{l}\mathrm{Rn} \\
12\end{array}$ & $\bar{T}$ & 18 & $\begin{array}{l}\mathrm{fr} \\
\mathrm{e} \\
\mathrm{s} \\
\mathrm{h} \\
\mathrm{l}\end{array}$ & $\begin{array}{l}\text { Fresh leaves are } \\
\text { crushed, water is added } \\
\text { and mixed with powdered } \\
\text { shell of snail }\end{array}$ & $\begin{array}{l}\text { Oral, } 1 / 2 \text { cup taken } 3 \times \text { per } \\
\text { day after meals }\end{array}$ & $\begin{array}{l}\text { (Muthukumaran et al, } \\
\text { 2011) }\end{array}$ & No report & $\begin{array}{l}\text { Ulcers, leprosy, } \\
\text { diabetes } \\
\text { (Subhadradevi et al., } \\
\text { 2011) }\end{array}$ \\
\hline $\begin{array}{l}\text { Senna petersiana } \\
\text { (Bolle) Lock, } \\
\text { Fabaceae }\end{array}$ & $\begin{array}{l}\text { Munemben } \\
\text { embe }\end{array}$ & $\mathrm{Rn} 2$ & $S$ & 27 & $\begin{array}{l}\mathrm{s} \\
\mathrm{e}\end{array}$ & $\begin{array}{l}\text { Dry seeds ground into } \\
\text { fine powder and boiled in } \\
\text { water }\end{array}$ & $\begin{array}{l}\text { Oral, } 1 / 2 \text { cup taken } 2 x \text { per } \\
\text { day after meals }\end{array}$ & Samie et al., 2010 & No report & $\begin{array}{l}\text { Venereal diseases } \\
\begin{array}{ll}\text { (Tshikalange et al., } \\
\text { 2005); } & \text { infertility } \\
\text { (Steenkamp, 2003) }\end{array}\end{array}$ \\
\hline $\begin{array}{l}\text { Strychnos } \\
\text { madagascariensis } \\
\text { Poir } \\
\text { Loganiaceae }\end{array}$ & Mukwakwa & $\begin{array}{l}\mathrm{Rn} \\
25\end{array}$ & $\mathrm{~T}$ & 18 & $\begin{array}{l}\text { st } \\
b\end{array}$ & $\begin{array}{l}\text { Ground into fine powder } \\
\text { and } \\
\text { boiled in water }\end{array}$ & $\begin{array}{l}\text { Oral, } 1 / 2 \text { cup is taken } 3 x \\
\text { per day for a week }\end{array}$ & No report & No report & $\begin{array}{l}\text { Fever (Ribeiro et al., } \\
\text { 2010), diarrhoea (de } \\
\text { Wet et al., 2010) }\end{array}$ \\
\hline $\begin{array}{l}\text { Solanum nigrum } \\
\text { L. } \\
\text { Solanaceae }\end{array}$ & Muxe & $\operatorname{Rn} 7$ & $\mathrm{H}$ & 27 & $f$ & $\begin{array}{l}\text { Green berries are } \\
\text { pounded, water is added } \\
\text { and is filtered }\end{array}$ & $\begin{array}{l}\text { A cupful of the juice is } \\
\text { taken orally } 3 x \text { per day }\end{array}$ & $\begin{array}{l}\text { (Mehjabeen et al., } \\
\text { 2011) }\end{array}$ & No report & $\begin{array}{l}\text { Ringworm (Jain et al., } \\
\text { 2011), Antiflammatory } \\
\text { (Ravi et al., 2009) }\end{array}$ \\
\hline $\begin{array}{l}\text { Solanum } \\
\text { panduriforme } \\
\text { Dunal, } \\
\text { Solanaceae }\end{array}$ & Ndhulwani & $\begin{array}{l}\mathrm{Rn} \\
19\end{array}$ & S & 18 & $f$ & $\begin{array}{l}\text { Dry fruits are burnt, } \\
\text { ashes mixed with animal } \\
\text { fat. }\end{array}$ & $\begin{array}{l}\text { Topical application on } \\
\text { the female genitals } 2 x \\
\text { per day for a week. }\end{array}$ & $\begin{array}{l}\text { No activity } \\
\text { (Steenkamp et al., } \\
\text { 2007) }\end{array}$ & No report & $\begin{array}{l}\text { Diarrhoea (McGaw et } \\
\text { al., 2008) }\end{array}$ \\
\hline $\begin{array}{l}\text { Strychnos } \\
\text { potatorum L.f. } \\
\text { Loganiaceae }\end{array}$ & $\begin{array}{l}\text { Mukongovh } \\
\text { oti }\end{array}$ & $\begin{array}{l}\mathrm{Rn} \\
34\end{array}$ & $\mathrm{~T}$ & 45 & $b$ & $\begin{array}{l}\text { Used together with the } \\
\text { roots of } A \text { senegalensis } \\
\text { and then soaked in cold } \\
\text { water for } 2 \text { days }\end{array}$ & $\begin{array}{l}\text { Maceration is used as } \\
\text { mouth wash or douche } \\
2 x \text { per day }\end{array}$ & No report & (Hamza et al., 2006) & $\begin{array}{l}\text { Leucoderma, diabetes } \\
\text { (Ekambaram et al., } \\
\text { 2010) }\end{array}$ \\
\hline $\begin{array}{l}\text { Tabernaemontana } \\
\text { elegans } \\
\text { Stapf, } \\
\text { Apocynaceae }\end{array}$ & Muhatu & Rn 1 & $\mathrm{~T}$ & 36 & $r$ & $\begin{array}{l}\text { Dry roots are pulverized } \\
\text { and boiled in water }\end{array}$ & $\begin{array}{l}\text { Decoction is taken orally, } \\
\text { a cupful } 3 x \text { per day }\end{array}$ & $\begin{array}{l}\text { (Steenkamp et al., } \\
\text { 2007) }\end{array}$ & No report & $\begin{array}{l}\text { Sexually transmitted } \\
\text { infections (De Wet et } \\
\text { al., 2012) }\end{array}$ \\
\hline $\begin{array}{l}\text { Tagetes minuta L. } \\
\text { Asteraceae }\end{array}$ & $\begin{array}{l}\text { Mushashth } \\
\text { uri }\end{array}$ & $\begin{array}{l}\mathrm{Rn} \\
20\end{array}$ & $\mathrm{H}$ & 27 & I & $\begin{array}{l}\text { Grind dry leaves and boil } \\
\text { in water }\end{array}$ & $\begin{array}{l}\text { Oral, } 1 \text { cup is taken while } \\
\text { still hot once per day }\end{array}$ & (Motsei et al., 2003) & $\begin{array}{l}\text { Oral candidiasis } \\
\text { (Hamza et al., 2006) }\end{array}$ & $\begin{array}{l}\text { Smallpox, earache } \\
\text { (Shahzadi et al., 2010) }\end{array}$ \\
\hline $\begin{array}{l}\text { Trimeria } \\
\text { grandifolia } \\
\text { (Hochst.) Warb. }\end{array}$ & Xidengani & $\operatorname{Rn} 4$ & $\mathrm{~T}$ & 9 & I & Chewing of fresh leaves & Oral, $3 x$ per day & No report & No report & $\begin{array}{l}\text { Heart burns (Okello et } \\
\text { al., 2010) }\end{array}$ \\
\hline
\end{tabular}




\begin{tabular}{|c|c|c|c|c|c|c|c|c|c|c|}
\hline $\begin{array}{l}\text { Flacourtiaceae } \\
\text { Vangueria } \\
\text { infausta Burch. } \\
\text { subsp. Infausta, } \\
\text { Rubiaceae }\end{array}$ & Muzwilu & $\begin{array}{l}\mathrm{Rn} \\
43\end{array}$ & $\mathrm{~T}$ & 45 & $\begin{array}{l}\mathrm{r} \\
\mathrm{b}\end{array}$ & $\begin{array}{l}\text { Chopped into small } \\
\text { pieces } \\
\text { and then boiled }\end{array}$ & $\begin{array}{l}\text { Oral, cupful decoction is } \\
\text { taken } 3 x \text { per day, also } \\
\text { used to treat diarrhoea }\end{array}$ & (de Boer et al., 2005) & $\begin{array}{l}\text { Oral candidiasis } \\
\text { (Chinsembu and } \\
\text { Hedimbi, 2010) }\end{array}$ & $\begin{array}{l}\text { Coughs (McGaw et al., } \\
\text { 2008) malaria, } \\
\text { pneumonia, (Venter } \\
\text { and Venter, 1996) }\end{array}$ \\
\hline $\begin{array}{l}\text { Warburgia } \\
\text { salutaris } \\
\text { (G.Bertol.) Chiov. } \\
\text { Canellaceae }\end{array}$ & Mulanga & $\begin{array}{l}\mathrm{Rn} \\
22\end{array}$ & $\mathrm{~T}$ & 36 & $\begin{array}{l}\text { ro } \\
\text { ot } \\
\mathrm{s}\end{array}$ & $\begin{array}{l}\text { Ground into powder, } 1 \\
\text { spoonful is added into } \\
\text { the } \\
\text { soft porridge }\end{array}$ & $\begin{array}{l}\text { Oral, twice per day, } \\
\text { Overdose causes } \\
\text { stomach ache }\end{array}$ & (Motsie et al., 2003). & $\begin{array}{l}\text { Thrush (van Wyk, } \\
\text { 2011) }\end{array}$ & $\begin{array}{l}\text { Malaria (Bussmann et } \\
\text { al., 2006), HIVIAIDS } \\
\text { (Larmorde et al., 2010) }\end{array}$ \\
\hline $\begin{array}{l}\text { Withania } \\
\text { somnifera (L.) } \\
\text { Dunal, } \\
\text { Solanaceae }\end{array}$ & $\begin{array}{l}\text { Musalamar } \\
\text { ubini }\end{array}$ & $\begin{array}{l}\mathrm{Rn} \\
33\end{array}$ & $S$ & 64 & $r$ & $\begin{array}{l}\text { Used together with few } \\
\text { leaves of Ensete } \\
\text { ventricosum which are } \\
\text { boiled together }\end{array}$ & $\begin{array}{l}\text { A cupful of decoction is } \\
\text { taken orally } 3 x \text { per day }\end{array}$ & $\begin{array}{l}\text { (Jain and Varshney, } \\
\text { 2011) }\end{array}$ & No report & $\begin{array}{l}\text { Cancer, fever } \\
\text { (Maregesi et al., } \\
\text { 2007), Diarrhoea } \\
\text { (McGaw et al., 2008) }\end{array}$ \\
\hline $\begin{array}{l}\text { Wrightia } \\
\text { natalensis Stapf. } \\
\text { Apocynaceae }\end{array}$ & Musunzi & $\begin{array}{l}\mathrm{Rn} \\
11\end{array}$ & $\mathrm{~T}$ & 27 & $\begin{array}{l}\mathrm{r} \\
\mathrm{b}\end{array}$ & $\begin{array}{l}\text { Ground into fine powder } \\
\text { and put into hot water }\end{array}$ & $\begin{array}{l}\text { Infusion is used as } \\
\text { gargle } 2 x \text { per day }\end{array}$ & No report & No report & $\begin{array}{l}\text { Gonorrhoea, } \\
\text { aphrodisiac (Obi et al., } \\
\text { 2002) }\end{array}$ \\
\hline
\end{tabular}

Key: $T=$ tree, $\mathrm{S}=$ shrub, $\mathrm{H}=$ =herb, l=leaves, $\mathrm{b}=$ =bark, $r=$ roots, $\mathrm{f}=$ =fruit, se=seeds, st=stem, wp=whole plant 
The most popular plants used by traditional healers were Hippocratea longipetiolata Oliv., Spilanthes acmella (L.) Murray, Schkuhria pinnata (Lam.) Kuntze ex Thell, Clerodendrum glabrum E.Mey, Osyris lanceolata Hochst. \& Steud., Faurea saligna Harv., Richardia brasiliensis Gomes, Withania somnifera (L.) Dunal, Elaeodendron transvaalense (Burtt Davy), Acacia caffra Thunb. Wild., Strychnos potatorum L.f., Schotia brachypetala Sond., Croton gratissimus Burch. and Vangueria infausta Burch. subsp. infausta, with their frequency index ranging from $45 \%$ to $73 \%$ (Table 1).

The fact that the same species are used by several traditional healers, and that several of these species are identified as medicinal plants from other communities in the literature provides confidence on their value. Furthermore, the use of the plant species by different cultural groups may also indicate their potential pharmacological efficacy. It is worth noting that 28 species, representing $62,2 \%$ of those recorded, have shown to have anti-Candida activity and 14 species $(31 \%)$ have been reported to have antifungal use elsewhere in the literature (Table 1). Only one plant species, Solanum panduriforme, has been reported to be inactive against Candida albicans (Table 2). However, this may be due to the fact that water was used as an extraction solvent while in the traditional preparation dried fruits are burnt and mixed with animal fat. This gives some credibility to the information we collected from the informants. Based on our literature search, 12 plant species claimed to be used to treat candidiasis and related fungal infections were recorded for the first time: Acacia caffra (Thunb) Wild., Amaranthus spinosus L, Cissampelos torulosa E.Mey, Faurea saligna Harv, Ozoroa engleri R. Fern. \& A. Fern, Pterocarpus rotundifolius Druce, Rinorea angustifolia Baill, Schkuhria pinnata (Lam.) Kuntze ex Thell, Strychnos madagascarienses Poir, Sophora microphylla Aiton, Trimeria grandifolia (Hochst.) Warb and Wrightia natalensis Stapf. These species have never been tested for anti-Candida activity

\subsection{Growth forms and plant parts used}

Twenty-three $(51,1 \%)$ tree species are highly used by traditional healers more than shrubs and herbs 33.3 and $15.5 \%$ respectively. This is because trees are available throughout the year as indicated by traditional practioners. . It is reported that trees usually bear greater quantities of compounds such as phenols, tannins, alkaloids, triterpenes and quinones than do shrubs and herbaceous species (Cartaxo et al., 2010), and this may also provide a reason why trees are preferred to other growth forms by the traditional healers.

The most widely used plant parts in the therapeutic preparations of remedies were roots and barks followed by roots and bark mixture ) (Fig 2 ). Several indigenous communities elsewhere also utilize mostly roots for the preparation of herbal remedies (Okello et al., 2010, Oyedemi et al., 2009; Appidi et al., 2008;). traditional healers prefer to use roots 
and bark because they believe that they have more healing powers than other plant parts. Studies by Maroyi, 2011 shows that roots and other underground parts have high concentrations of bioactive compounds. On the other hand there were no statistically significant antimicrobial activity differences between leaf and bark extracts of Marula (Eloff, 2001). Worthy of note is that plant species in the area under study are frequently exposed to long periods of drought and they shed their leaves, so this may be an additional reason why roots and barks are preferred. According to the informants, plant materials are mainly collected in the winter season and time of collection is early in the morning. They believe that plants absorb plenty of water during the summer rainfall that may dilute the active principles, resulting in the loss of healing power of the remedy. In a study of the variation in antibacterial activity of Schotia brachypetala ethanol and water leaf extracts, McGaw et al. (2002) reported that monthly variation in activity against a panel of bacterial species was not marked, although there appeared to be a tendency towards slightly higher activity in the summer months.

Frequent harvesting of roots and bark has a negative influence on the survival of plants, and is therefore discouraged. Some studies (Shai et al., 2009, Eloff, 2001) have shown that plant leaf extracts are as active or even more active than extracts of other plant parts. Thus, to foster sustainability, traditional healers should be encouraged to use plant leaves. However, this might only be valid if the chemistry of the roots or bark is similar to that of the leaves. There are several communities in Africa and elsewhere who are mainly using leaves to treat a variety of ailments, examples are Burkina Faso (Nadembega et al., 2011), Cameroon (Telefo et al., 2011), , Uganda (Ssegawa and Kasenene, 2007) India (Namsa et al., 2009)

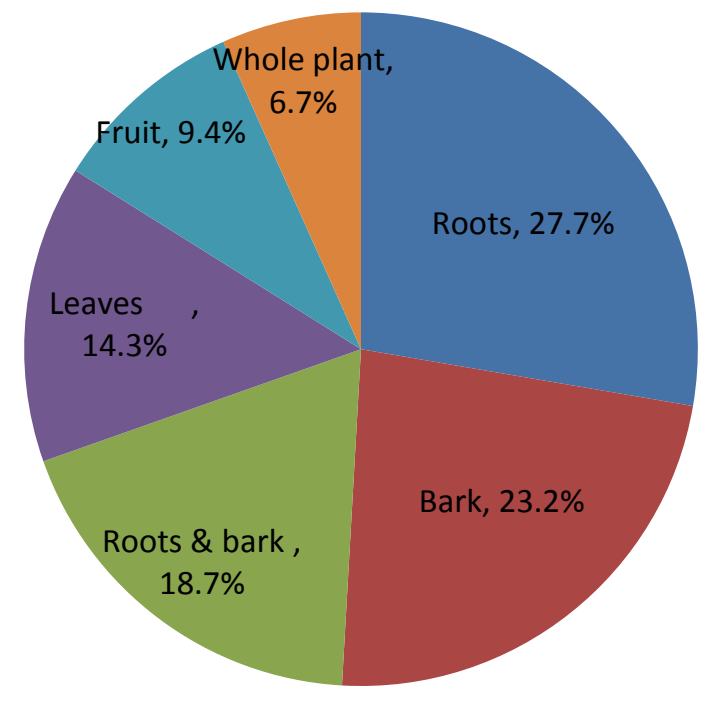

Fig. 2. Percentage of plant parts used for preparing the traditional remedies 
3.4. Preparation, dosage and route of administration of the remedy.

The most frequently used methods in the preparation of the herbal remedies were decoctions followed by infusions macerations and burning). The use of decoctions and infusions as the methods of choice is also supported by other studies (Keter and Mutiso, 2012, Namukobe et al., 2011,.

With regard to burning, plant materials such as roots and barks are burnt to a certain degree, and then the fire is doused so that they do not become ash lest they lose their healing properties. In some plant species such as Dichrostachys cinerea, dry splinter of wood is burnt at one end, liquid that oozes out is used as a remedy for treating fungal infections.

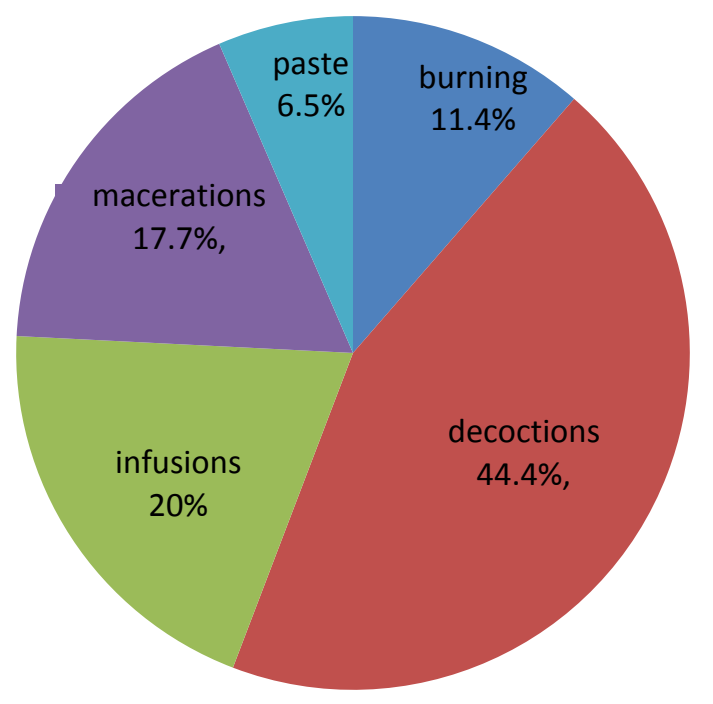

Fig. 3. Reported methods of preparing the traditional remedies in percentage.

The majority of these preparations were constituted by single plant species (84\%) and $16 \%$ was constituted from multiple plant species and similar findings were reported by other researchers (Packer et al., 2012, Ranganathan et al., 2012,). Most traditional healers reported that multiple preparations of the remedy are normally administered to the patient if the disease is severe. There was a consensus among the traditional healers that some plants enhance the action of others, powdered roots of $E$ transvaalense were reported to be added to most of the herbal preparations. transvaalense is reported as having antiviral and anticandidal properties (Samie et al., 2010 and Bessong et al., 2005). 
We also found that dosage was estimated using either lids, spoons, cups, pinches or handfuls. It was difficult to get the information on exactly how much plant material was used to make the extracts. In most cases dosage was determined according to the severity of the disease and age of the patientHowever, there was a lack of precision in the determination of the dosage to be taken by the patient. McGaw et al. (2007) reported that drawbacks of traditional medicinal plant remedies include uncertain dosages and lack of standardization.

Traditional healers reported that the prepared therapeutic remedies may be stored in powder form, especially for scarce medicinal plants, and liquid remedies (decoctions or infusions) are prepared and administered to patients on a daily basis. The therapeutic preparations are either stored in dried horns or skins of animals, calabashes, clay pots orwooden bottles. The use of either tins, plastics or glass bottles is discouraged because they absorb heat which may destroy the healing properties of the remedy. Unprocessed plant materials such as roots, fruit and stem bark are stored in dried form for future use in the traditional surgery which has a thatched roof.

With regard to the administration routes of the remedies, four main routes were reported, namely douche (20\%), oral (56\%), mouthwash (13,3\%) and topical (10,7\%). However, oral application was the most commonly used route of administration. This is in agreement with the results of various ethnobotanical studies elsewhere (Mukazayire et al., 2011; Philander et al., 2011, Wambugu et al., 2011;). The use of the oral route of administration did not come as a surprise because this is in line with orthodox medicine where the preferred route is frequently oral. Most of these remedies were taken with some food twice or three times per day and some additives such as fats were also used, especially for topical application.

\subsection{Conservation status of medicinal plants}

The main factors threatening the conservation status of medicinal plants recorded during the interviews were unsustainable methods of harvesting, logging for firewood, use of plants for building materials, crafts and current agricultural trends. However, the informants stressed that they practice sustainable methods of harvesting the medicinal plants from the wild because they know that these plants are their primary source of income. In some of the instances, before the collection, they conduct some rituals such as sprinkling of snuff, bowing, invoking of their ancestors, etc. The informants blamed herbalists for the destruction of the medicinal plants.

Some of the harvesting techniques employed by the traditional healers included stripping of bark from the western and eastern sides of the tree to avoid ring barking of the stem; when collecting the whole plant, they ensured that some individuals remained behind; when collecting roots, side roots were collected, leaving the main ones for the plant to survive etc. The informants believed that if the plant from which they collected the plant material dies, then 
the patient would not be cured by the remedy. This is another way of instilling sustainable methods of harvesting plant material, resulting in the conservation of the medicinal plantsThe present inventory represents a contribution of the natural flora of this area to a global approach in controlling candidiasis and related infections.

\section{Conclusion}

A total of 45 plant species used by the local traditional practitioners to manage candidiasis and related fungal infections were documented. The results of this study have shown that traditional medicine still plays an important role in meeting the primary health care needs of rural people in the area and that the information has a good evidential base. We reported for the first time 12 plants claimed to be used to treat candidiasis, and related fungal infections. This baseline study could help in identifying plant species for in depth investigation in order to establish their claimed potential in the management of candidiasis and related fungal infections. As a follow up on this work research on Clerodendrum glabrum one of the species identified, have led to the isolation and characterization of clerodendrumic acid, a new triterpenoid with antimicrobial activity (Masevhe et al., 2013).

\section{Acknowledgements}

We are indebted to the traditional healers for sharing with us their valuable knowledge on medicinal plants of the area. Worthy of special mention include Munyai T, Munyadziwa $\mathrm{P}$ and Mudau $\mathrm{F}$ for their immense contribution in this study. Financial support was provided by the Netherlands Universities Foundation for International Cooperation (NUFFIC), the National Research Foundation (NRF- IFR2011041300066), The Medical Research Council (SIR JNEloff), University of Pretoria and University of Venda. We are grateful to SANBI (South African National Biodiversity Institute) for the authentication of the identities of the collected plants..

\section{References}

Abbasi, A.M., Khan, M.A., Ahmad, M., Zafar, M., Jahan, S., Sultana, S., 2010. Ethnopharmacological application of medicinal plants to cure skin diseases and in folk cosmetics among the tribal communities of North-West Frontier Province, Pakistan. Journal of Ethnopharmacology 128, 322-335.

Acocks, J.P.H., 1988. Veld types of South Africa, $3^{\text {rd }}$ edition. Botanical Research Institute, South Africa.

Adamu, H.M., Abayeh, O.J., Agho, M.O., Abdullahi, A.L., Uba, A., Dukku, H.U., Wufem, B.M., 2005. An ethnobotanical survey of Bauchi State herbal plants and their antimicrobial activity. Journal of Ethnopharmacology 99, 1-4.

Adekunle, A.A., 2000. Antifungal property of crude extracts of Brachystigia eurycoma and Richardia. brasiliensis. Nigerian 
Journal of Natural Products and Medicine 4, 70-72.

Ajibesin, K.K., Ekpo, B.A., Bala, D.N., Essien, E.E., Adesanya, S.A., 2008. Ethnobotanical survey of Akwa Ibom State of Nigeria. Journal of Ethnopharmacology 115, 387-408.

Appidi, J.R., Grierson, D.S., Afolayan, A.J., 2008. Ethnobotanical study of plants used in the treatment of diarrhea in the Eastern Cape, South Africa. Parkistan Journal of Biological Sciences 11, 1961-1963.

Arabi, Z., Sardari, S., 2010. An investigation into the antifungal property of Fabaceae using bioinformatics tools. Avicenna Journal of Medical Biotechnology 2, 93-100.

Aref , H.L., Salah, K.B.H., Chaumont, J.P., Fekih, A., Aouni, M., Said, K., 2010. In vitro antimicrobial activity of four Ficus carica latex fractions against resistant human pathogens (antimicrobial activity of Ficus carica latex). Pakistan Journal of Pharmaceutical Science 23, 53-58.

Arnold, H.J., Gulumiam, M., 1984. Pharmacopoeia of traditional medicine in Venda. Journal of Ethnopharmacology 12, 35-74.

Bekalo, T.H., Woodmatas, S.D., Woldemariam, Z.A., 2009. An ethnobotanical study of medicinal plants used by local people in the lowlands of Konta Special Woreda, southern nations, nationalities and peoples regional state, Ethiopia. Journal of Ethnobiology and Ethnomedicine 5, 26-33.

Bessong, P.O., Obi, C.L., Andreola, M.L., Rojas, L.B., Pouysegu, L., Igumbor, E., Meyer, J.J.M., Quideau, S., Litvak, S., 2005. Evaluation of selected South African medicinal plants for inhibitory properties against human immunodeficiency virus type 1 reverse transcriptase and integrase. Journal of Ethnopharmacology 99, 83-91.

Bisi-Johnson, M. A., Obi, C. L., Kambizi, L., Nkomo, M., 2010. A survey of indigenous herbal diarrhoeal remedies of O.R. Tambo district, Eastern Cape Province, South Africa. African Journal of Biotechnology 9,1245-1254.

Bornman, M., Schlemmer, L., van der Walt, T., van Dyk, C., Bouwman, H., 2012. Implications for health education and intervention strategies arising from children's caregivers concerns following successful malaria control. Transactions of the Royal Society of Tropical Medicine and Hygiene 106,408- 414.

Bussmann, R.W., Sharon, D., 2006. Traditional plant use in Northern Peru, tracking two thousand years of health culture. Journal of Ethnobiology and Ethnomedicine 2, 47.

Buwa, L.V., van Staden., J., 2006. Antibacterial and antifungal activity of traditional medicinal plants used against venereal diseases in South Africa. Journal of Ethnopharmacology 103, 139-142.

Buwa, L.V.,Van Staden, J., 2007. Effects of collection time on the antimicrobial activities of Harpephyllum caffrum bark. South African Journal of Botany 73, 242-247.

Camejo-Rodrigues, J., Ascensao, L., Bonet, M.A., Vallès, J., 2003. An ethnobotanical study of medicinal and aromatic plants in the Natural Park of "Serra de São Mamede" (Portugal). Journal of Ethnopharmacology 89, 199-209.

Cartaxo, S.L., Souza, M.M.A., de Albuquerque, U.P., 2010. Medicinal plants with bioprospecting potential used in semi-arid northeastern Brazil. Journal of Ethnopharmacology 131, 326-342.

Chahal, J., Ohlyan, R., Kandale, A., Walia, A., Sidharth, P., 2011. Introduction, phytochemistry, traditional uses and biological 
activity of genus Piper: A review. International Journal of Current Pharmaceutical Review and Research 2, 131-144.

Chinsembu, K.C., Hedimbi, M., 2010. An ethnobotanical survey of plants used to manage HIVIAIDS opportunistic infections in Katima Mulilo, Caprivi region, Namibia. Journal of Ethnobiology and Ethnomedicine 6, 25-34.

De Boer, H.J., Kool, A., Broberg, A., William, R., Mziray, W.R., Hedberg, I., Levenforsd, J.J., 2005. Anti-fungal and antibacterial activity of some herbal remedies from Tanzania. Journal of Ethnopharmacology 96, 461-469.

De Wet, H., Nzama, V.N., Van Vuuren, S.F., 2012. Medicinal plants used for the treatment of sexually transmitted infections by lay people in northern Maputaland, KwaZulu-Natal Province, South Africa. South African Journal of Botany 78, $12-20$.

De Wet, H., Nkwanyana, M.N., Van Vuuren, S.F., 2010. Medicinal plants used for the treatment of diarrhoea in northern Maputaland, KwaZulu-Natal Province, South Africa. Journal of Ethnopharmacology 130, 284-289.

De Wet, H., Van Wyk, B.E., 2008. An ethnobotanical survey of Southern African Menispermaceae. South African Journal of Botany 74, 2-9.

Drewes, S.E., Mashimbye, M.J., Frix, J.S., Ramesar, N.Y., 1991. 11,I I-Dimethyl-I,3,8,IO-tetrahydroxy-9-methoxypeltogynan and three pentacyclic triterpenes from Cassine transvaalensis. Phytochemistry 30, 3490 -3493.

Ekambaram, S., Perumal S.S., Subramanian, V., 2010. Evaluation of antiarthritic activity of Strychnos potatorum Linn seeds in Freund's adjuvant induced arthritic rat model. BMC Complementary and Alternative Medicine 10, 56-65.

Eloff, J.N., 2001. Antibacterial activity of Marula (Sclerocarya birrea (A. rich.) Hochst. subsp. caffra (Sond.) Kokwaro) (Anacardiaceae) bark and leaves. Journal of Ethnopharmacology 76, 305-308.

Eloff, J.N., famakin, J.O., katerere, D.R.P., 2005 Combretum woodii (Combretaceae) leaf extracts have high activity against Gram-negative and Gram-positive bacteria. African Journal of Biotechnology 4, 1161-116.

Gessler, M.C., Msuya, D.E., Nkunya, M.H.H., Mwasumbi, L.B., Schar, L.B., Heinrich, M, Tanner, M., 1995.Traditional healers in Tanzania: the treatment of malaria with plant remedies. Journal of Ethnopharmacology 48,13 I-144.

Graham, G., Quinn, M.L., Fabricant, D.S., Farnsworth, N.R., 2000. Plants used against cancer-an extension of the work of Jonathan Hartwell. Journal of Ethnopharmacology 73, 347-377.

Grant R., Thomas V., 2000. Sappi tree spotting. Jacana, Johannesburg.

Guimarães, R., Barros, L., Carvalho, A.M., 2011. Infusions and decoctions of mixed herbs used in folk medicine: synergism in antioxidant potential. Phytotherapy. Wiley Online Library.

Hamill, F.A., Apio S., Mubiru, N.K., Mosango, M., Bukenya-Ziraba, R., Maganyi, O.W., Soejarto, D.D., 2000. Traditional herbal drugs of southern Uganda 1. Journal of Ethnopharmacology 70, 281- 300.

Hamza, O.J.M., Van den Bout-van den Beukel, C.J.P., Matee, M.I.N., Moshi, M.J., Mikx, F.H.M., Selemani, H.O., Mbwambo, Z.H., Van der Ven, A.J.A.M., Verweij, P.E., 2006. Antifungal activity of some Tanzanian plants used traditionally for the treatment of fungal infections. Journal of Ethnopharmacology 108, 124-132.

Hutchings, A., Scott, A.H., Lewis, G., Cunningham, A., 1996. An inventory of Zulu medicinal plants. University of Natal, Pietermaritzburg. 
Idowu, O.A., Soniran, O.T., Ajana, O., Aworinde, D.O., 2010. Ethnobotanical survey of antimalarial plants used in Ogun State, Southwest Nigeria. African Journal of Pharmacy and Pharmacology 4, 55-60.

Jain, P., Varshney, R., 2011. Antimicrobial activity of aqueous and methanolic extracts of Withania somnifera (Ashwagandha). Journal of Chemical and Pharmaceutical Research 3, 260-263.

Jeong, M.R., Kim, H.Y., Cha, J.D., 2009. Antimicrobial activity of methanol extract from Ficus carica leaves against oral bacteria. Journal of Bacteriology and Virology 39, 97- 102.

Kambizi, L., Afolayan, A.J., 2001. An ethnobotanical study of plants used for the treatment of sexually transmitted diseases in Guruve District, Zimbabwe. Journal of Ethnopharmacology 77, 5-9.

Keter, L.K., Mutiso, P.C., 2012. Ethnobotanical studies of medicinal plants used by Traditional Health Practitioners in the management of diabetes in Lower Eastern Province, Kenya. Journal of Ethnopharmacology 139, 74- 80.

Kumar, B.S.A., Lakshman, K., Jayaveera, K.N., 2011. Comparative antipyretic activity of methanolic extracts of some species of Amaranthus. Asian Pacific Journal of Tropical Biomedicine S, 47-50.

Lahiff, E., 1997. Land, water and local governance in South Africa: a case study of the Mutale river valley. Rural resources rural livelihoods working paper series.

Lamorde, M.,Tabuti, J.R.S., Obua, C., Kukunda-Byobona, C., Lanyero, H., Byakika-Kibwikaa, P., Bbosa, G.S., Lubega, A., Ogwal-Okeng, J., Ryan, M., Waako, P.J., Merry, C., 2010. Medicinal plants used by traditional medicine practitioners for the treatment of HIVIAIDS and related conditions in Uganda. Journal of Ethnopharmacology 130, 43-53.

Libman, A., Bouamanivong, S., Southavong, B., Sydara, K., Soejarto, D.D., 2006. Medicinal plants: An important asset to health care in a region of Central Laos. Journal of Ethnopharmacology 106, 303-311.

Lulekal, E., Kelbessa, E., Bekele, T., Yineger, H., 2008. An ethnobotanical study of medicinal plants in Mana Angetu District, south eastern Ethiopia. Journal of Ethnobiology and Ethnomedicine 4, 1-10.

Mabogo, D.E.N., 1990. The ethnobotany of the Vhavenda. Unpublished MSc Thesis, University of Pretoria, Pretoria.

Madikizela, B., Ndhlala, A.R., Finnie, J.F., Van Staden, J., 2012. Ethnopharmacological study of plants from Pondoland used against diarrhoea. Journal of Ethnopharmacology 141, 61-71.

Magassouba, F.B., Diallo, A., Kouyate, M., Mara F., Mara, O., Bangoura, O., Camara, A., Traore, S., Diallo, A.K., Zaoro, M., Lamah, K., Diallo, S., Camara, G., Maregesi, S.M., Ngassapa, O.D., Pieters, L., Vlietinck, A.J., 2007. Ethnopharmacological survey of the Bunda district, Tanzania: Plants used to treat infectious diseases. Journal of Ethnopharmacology113, 457-470.

Mahwasane, S.T., Middleton, L., Boaduo, N., 2013. An ethnobotanical survey of indigenous knowledge on medicinal plants used by the traditional healers of the Lwamondo area, Limpopo province, South Africa. South African Journal of Botany 88, 69-75.

Maregesi, S.M., Ngassapa, O.D., Pieters, L., Vlietinck, A.J., 2007. Ethnopharmacological survey of the Bunda district, Tanzania, plants used to treat infectious diseases. Journal of Ethnopharmacology 113, 457-470. 
Maroyi, A., 2011. An ethnobotanical survey of medicinal plants used by the people in Nhema communal area, Zimbabwe. Journal of Ethnopharmacology 136, 347-354.

Masevhe, N.A., awouafack, M,, Ahmed, A.S,, Mcgaw, L.J,, Eloff, J.N., 2013. Clerodendrumic acid, a new triterpenoid from Clerodendrum glabrum (Verbenaceae) and antimicrobial activity of fractions and constituents. Helvetica Chemica Acta 63, 1693-1703.

Mbeunkui, F., Grace, M.H., Lategan, C., Smith, P.J., Raskin I., Lil, M.A., 2011.Isolation and identification of antiplasmodial Nalkylamides from Spilanthes acmella flowers using centrifugal partition chromatography. Journal of Chromatography B 879, 1886- 1892.

McGaw, L.J., van der Merwe, D., Eloff, J.N., 2007. In vitro anthelmintic, antibacterial and cytotoxic effects of extracts from plants used in South African ethnoveterinary medicine. The Veterinary Journal 173, 366-372.

McGaw, L.J., Jager, A.K., Van Staden, J., 2000. Antibacterial, anthelmintic and anti-amoebic activity in South African medicinal plants. Journal of Ethnopharmacology 72, 247-263.

McGaw, L.J., Jager, A.K., Van Staden, J., 2002. Variation in antibacterial activity of Schotia species. South African Journal of Botany 68, 41-46.

McGaw, L.J., Lall, N., Meyer, J.J.M., Eloff, J.N., 2008. The potential of South African plants against Mycobacterium infections. Journal of Ethnopharmacology 119, 482-500.

Mehjabeen, A.M., Jahan, N., Zia-Ul-Haq, M., Alam, S.M., Wazir, A., Hassan, S., 2011. Antimicrobial screening of some plants of medicinal importance. Pakstan Journal of Botany.43, 1773-1775.

Meyer, J.J.M., Rakuambo, N.C., Hussein, A.A., 2008. Novel xanthones from Securidaca longepedunculata with activity against erectile dysfunction. Journal of Ethnopharmacology 119, 599-603.

Motsei, M.L., Lindsey, K.L., Van Staden, J., Jager, A.K., 2003. Screening of traditionally used South African plants for antifungal activity against Candida albicans. Journal of Ethnopharmacology 86, 235-241.

Mukazayire, M.J., Minani, V., Ruffo, C.K., Bizuru, E., Stevigny, C., Duez, P., 2011.Traditional phytotherapy remedies used in Southern Rwanda for the treatment of liver diseases. Journal of Ethnopharmacology 138, 415-431.

Mulaudzi, R.B., Ndhlala, A.R., Kulkarni, M.G., Finnie, J.F., Van Staden, J. 2013. Antiinflammatoryandmutagenicevaluationofmedicinalplantsusedby Venda peopleagainstvenerealandrelateddiseases. Journal of Ethnopharmacology 146, 173-179.

Mulaudzi, R.B., Ndhlala, A.R., Kulkarni, M.G., Finnie, J.F., Van Staden, J., 2011. Antimicrobial properties and phenolic contents of medicinal plants used by the Venda people for conditions related to venereal diseases. Journal of Ethnopharmacology 135, 330-337.

Mulaudzi, R.B., Ndhlala, A.R., Kulkarni, M.G., Van Staden, J., 2012. Pharmacological properties and protein binding capacity of phenolic extracts of some Venda medicinal plants used against cough and fever. Journal of Ethnopharmacology 143, 185-93.

Muthee, J.K., Gakuya, D.W., Mbaria, J.M., Kareru, P.G., Mulei, C.M., Njonge, F.K., 2011. Ethnobotanical study of anthelmintic 
and other medicinal plants traditionally used in Loitoktok district of Kenya. Journal of Ethnopharmacology 135, 15-21. Muthukumaran, M., Elayarani, P., Shanmuganathan, P., Cholarajan, A., 2011. Antimicrobial activities of Cassia auriculata L and Morinda tinctoria Roxb. International Journal of Research in Pure and Applied Microbiology 1, 9-12.

Mzezewa, J., Misi, T., van Rensburg, L.D. 2010. Characterisation of rainfall at a semi-arid ecotope in the Limpopo Province (South Africa) and its implications for sustainable crop production. Water South Africa 36, 29-26.

Nadembega, P., Boussim, J.I., Nikiema, J.B., Poli, F., Antognoni, F., 2011. Medicinal plants in Baskoure, Kourittenga Province, Burkina Faso: An ethnobotanical study. Journal of Ethnopharmacology 133, 378-395.

Namsa, N.D., Tag, H., Mandal, M., Kalita, P., Das, A.K., 2009. An ethnobotanical study of traditional anti-inflammatory plants used by the Lohit community of Arunachal Pradesh, India. Journal of Ethnopharmacology 125, 234-245.

Namukobe, J., Kasenene, J.M., Kiremire, B.T., Byamukama, R., Kamatenesi-Mugisha, M., Krief, S., Dumontet, V., Kabasa, J.D., 2011. Traditional plants used for medicinal purposes by local communities around the Northern sector of Kibale National Park, Uganda. Journal of Ethnopharmacology 136, 236-245.

Obi, C.L., Potgieter, N., Randima, L.P., Mavhungu, N.J., Musie, E., Bessong, P.O., Mabogo, D.E.N., Mashimbye, J., 2002. Antibacterial activities of five plants against some medically significant human bacteria. South African Journal of Science 98, 25-28.

Offiah, N.V., Makama, S., Elisha, I.L., Makoshi, M.S., Gotep, J.G., Dawurung, C.J., Oladipo, O.O., Lohlum, A.S., Shamaki, D., 2011. Ethnobotanical survey of medicinal plants used in the treatment of animal diarrhoea in Plateau State, Nigeria. BMC Veterinary Research 7,36-49.

Otsuki, N., Dang, N.H., Kumagai, E., Kondo, A., Iwata, S., Morimoto, C., 2010. Aqueous extract of Carica papaya leaves exhibits anti-tumor activity and immunomodulatory effects. Journal of Ethnopharmacology 127, 760-767.

Oyedemi, S.O., Bradley G., Afolayan, A.J., 2009. Ethnobotanical survey of medicinal plants used for the management of diabetes mellitus in the Nkonkobe municipality of South Africa. Journal of Medicinal Plants Research 3, 1040 -1044.

Packer, J., Brouwer,N., Harrington, D., Gaikwad, J., Heron, R., Ranganathan, S., Vemulpad, S., Jamie J., 2012. An ethnobotanical study of medicinal plants used by the Yaegl Aboriginal community in northern New South Wales, Australia. Journal of Ethnopharmacology 139, 244 - 255.

Patel, M., Coogan, M.M., 2008. Antifungal activity of the plant Dodonaea viscosa var. angustifolia on Candida albicans from HIV-infected patients. Journal of Ethnopharmacology 118,173-176.

Pfaller, M.A., Diekema, D.J. 2007. Epidemiology of Invasive Candidiasis: a Persistent Public Health Problem. Clinical Microbiology Reviews 20, 133-163.

Pfoze, N.L., Yogendra, K., Bekington, M., 2012. Survey and assessment of ethnomedicinal plants used in Senapati District of Manipur State, Northeast India. Phytopharmacology 2, 285-311.

Philander, L. A., 2011. An ethnobotany of Western Cape Rasta bush medicine. Journal of Ethnopharmacology 138, 578 - 594.

Pinto, D.S., Tomaz, A.C.A., Tavares, J.F., Tenorio-Souza, F.H., Dias, C.S., Braz-Filho, R., Da-Cunha, E.V.L., 2008. 
Secondary metabolites isolated from Richardia brasiliensis Gomes (Rubiaceae). Brazilian Journal of Pharmacognosy 18, 367-372.

Ranganathan, R., Vijayalakshmi, R., Parameswari, P., 2012. Ethnomedicinal survey of Jawadhu hills in Tamil Nadu. Asian Journal of Pharmaceutical and Clinical Research 5, 45-49.

Ravi, V., Saleem, T.S.M., Patel, S.S., Raamamurthy, J., Gauthaman, K., 2009. Anti-Inflammatory effect of methanolic extract of Solanum nigrum Linn Berries. International Journal of Applied Research in Natural Products 2, 33-36.

Reichart, P.A., 2003.Oral manifestations in HIV infection: fungal and bacterial infections, Kaposi's sarcoma. Medical Microbiology Immunology 192, 165-169.

Ribeiro, A., Romeiras, M.M., Tavares, J., Faria, M.T., 2010. Ethnobotanical survey in Canhane village, district of Massingir, Mozambique: medicinal plants and traditional knowledge. Journal of Ethnobiology and Ethnomedicine 6, 33-48.

Rosas-Pinon, Y., Mejía, A., Diaz-Ruiz, G., Aguilar, M.I., Sanchez-Nieto, S., Rivero-Cruz, J.F., 2012. Ethnobotanical survey and antibacterial activity of plants used in the Altiplane region of Mexico for the treatment of oral cavity infections. Journal of Ethnopharmacology 141, 860-865.

Runyoro, D.K.B., Ngassapa, O.D., Matee M.I.N., Joseph, C.C., Moshi, M.J., 2006. Medicinal plants used by Tanzanian traditional healers in the management of Candida infections. Journal of Ethnopharmacology 106, 158 -165.

Samie, A., Tambani, T., Harshfield, E., Green, E., Ramalivhana J.N., Bessong, P.O., 2010. Antifungal activities of selected Venda medicinal plants against Candida albicans, Candida krusei and Cryptococcus neoformans isolated from South African AIDS patients. African Journal Biotechnology 9, 2965-2976.

Shahzadi, I., Hassan, A., Khan, U.W., Shah, M.M., 2010. Evaluating biological activities of the seed extracts from Tagetes minuta L. found in Northern Pakistan. Journal of Medicinal Plants Research 4, 2108-2112.

Shai, L.J, McGaw, L.J., Eloff, J.N., 2009. Extracts of the leaves and twigs of the threatened tree Curtisia dentate (Cornaceae) are more active against Candida albicans and other microorganisms than the stem bark extract. South African Journal of Botany 75, 363-366.

Singh, S., Prasad, R., Pathania, K., Joshi, H., 2012. Antifungal activity of Plumbagin and Isodiospyrin from Diospyros kaki root bark. . Asian Journal of Plant Science and Research 2, 1-5.

Ssegawa, P., Kasenene, J.M., 2007. Medicinal plant diversity and uses in the Sango bay area, Southern Uganda. Journal of Ethnopharmacology 113, 521-540.

Stafford, G.I., Pedersen, M.E., Van Staden, J., Jager, A.K., 2008. Review on plants with CNS-effects used in traditional South African medicine against mental diseases. Journal of Ethnopharmacology 119, 513- 537.

Steenkamp, V., 2003. Traditional herbal remedies used by South African women for gynaecological complaints. Journal of Ethnopharmacology 86, 97-108.

Steenkamp, V., Fernandes, A.C., Van Rensburg, C.E.J., 2007. Screening of Venda medicinal plants for antifungal activity against Candida albicans. South African Journal of Botany 73, 256-258. 
Subhadradevi, V., Asokkumar, K., Umamaheswari, M., Sivashanmugam, A.T., Ushanandhini J.R., Jagannath, P., 2011. Antimicrobial Activity of leaves and flowers of Cassia auriculata linn. Bangladesh Journal of Science and Industrial Research 46, 513-518.

Taylor, J.L.S., Rabe, T., McGaw, L.J., Jager, A.K., van Staden, J., 2001. Towards the scientific validation of traditional medicinal plants. Plant Growth Regulation 34, 23-37.

Telefo, P.B., Lienou, L.L.,Yemele, M.D., Lemfack, M.C., Mouokeu, C., Goka, C.S., Tagne, S.R., Moundipa, F.P., 2011. Ethnopharmacological survey of plants used for the treatment of female infertility in Baham, Cameroon. Journal of Ethnopharmacology 136, 178-187.

Thring, T.S.A., Weitz F.M., 2006. Medicinal plant use in the Bredasdorp/Elim region of the Southern Overberg in the Western Cape Province of South Africa. Journal of Ethnopharmacology 103, 261-275.

Tshikalange, T.E., Meyer J.J.M., Hussein A.A., 2005. Antimicrobial activity, toxicity and the isolation of a bioactive compound from plants used to treat sexually transmitted diseases. Journal of Ethnopharmacology 96, 515-519.

UNAIDS. 2011. World AIDS day report. Geneva, Switzerland.

Van Vuuren, S.F., Naidoo, D., 2010. An antimicrobial investigation of plants used traditionally in Southern Africa to treat sexually transmitted infections. Journal of Ethnopharmacology 130, 552- 558.

Van Vuuren, S.F., Viljoen, A.M., 2008. In vitro evidence of phyto-synergy for plant part combinations of Croton gratissimus (Euphorbiaceae) used in African traditional healing. Journal of Ethnopharmacology 119, 700-704.

Van Wyk, B.E., 2011. The potential of South African plants in the development of new medicinal products. South African Journal of Botany 77, 812-829.

Van Wyk, B.E.; Gericke, N. 2000. People's plants. Briza, South Africa.

Venter, A.,Venter, J.A., 1996. Making the most of indigenous trees. Briza, South Africa

Vermani, K., Garg, S., 2002. Herbal medicines for sexually transmitted diseases and AIDS. Journal of Ethnopharmacology 80, 49-66.

Wambugu, S.N., Mathiu, P.M., Gakuya, D.W., Kanui, T.I., Kabasa, J.D., Kiama, S.G., 2011. Medicinal plants used in the management of chronic joint pains in Machakos and Makueni counties, Kenya. Journal of Ethnopharmacology 137, 945- 955.

Zerabruk, S., Yirga, G., 2012. Traditional knowledge of medicinal plants in Gindeberet district, Western Ethiopia. South African Journal of Botany 78, $165-169$. 


\section{Questionnaire}

Age:

Gender:

Physical address and contact no.:

Education:

1. Information about the disease

Do you treat people with the following symptoms?

- mouth ulcers

- genital ulcers

- sore throat

- $\quad$ itching of genitals

- white discharge

2. Information about the medicinal plants

- Which plants do you use to treat candidiasis?

- Can you share with us information on plant parts used, method of preparation, storage of the remedy and dosage?

- Where do you collect your plants? Are you willing to show us the plants?

- Can you share with us some of your harvesting techniques?

- Do you conduct some rituals before the collection?

- What are the factors threatening the conservation status of the plants?

\begin{tabular}{|l|l|l|l|l|l|l|}
\hline Local name & Habit & Citation & Part used & $\begin{array}{l}\text { Method of } \\
\text { preparation }\end{array}$ & $\begin{array}{l}\text { Route of } \\
\text { administration }\end{array}$ & Dosage \\
\hline & & & & & & \\
\hline & & & & & & \\
\hline & & & & & & \\
\hline
\end{tabular}

